\title{
Construction of Robust Lyapunov Functions for Reaction Networks
}

\author{
M. Ali Al-Radhawi and David Angeli
}

\begin{abstract}
Although Chemical Reaction Networks (CRNs) form a rich class of nonlinear systems that can exhibit wide range of nonlinear behaviours, many common examples are observed to be asymptotically stable regardless of the kinetics. This paper presents the recently uncovered class of Graphically Stable Networks (GSNs) which is characterized by the existence of a robust Lyapunov function defined in the reaction coordinates. Subject to mild conditions, the existence of these functions guarantees asymptotic stability of a network regardless of the specific form of kinetics. Construction methods for these functions are provided and illustrated by examples.
\end{abstract}

\section{INTRODUCTION}

Chemical Reaction Networks (CRNs) are used as models in several areas of science and engineering; including chemical engineering, population dynamics, and molecular systems biology. The last application has drawn the recent interest of the systems and control community to this field [1], [2].

The central dilemma in this field is that the kinetic modeling required to construct a full mathematical model is highly uncertain and scarce. This is unlike the wealth of information available on the graphical description of these networks. Hence, the main aim has been to draw conclusions about the asymptotic behaviour of a network regardless of its kinetics. This has been advocated towards "complex biology without parameters" [3]. The presumed feasibility of this goal has been motivated by the observation that large classes of CRNs converge asymptotically to steady states regardless of the model of kinetics involved. Indeed, partial success has been achieved in this pursuit. The class of weakly reversible zero-deficiency networks with Mass-Action kinetics has been shown to have unique asymptotically stable equilibria in the interior of the orthant [4], [5], [1]. Monomolecular networks have also been handled within the framework of compartmental systems [6]. Recently, the monotonicity concept has been applied to provide graphical conditions for global convergence of some of these networks [7].

This paper reviews the recent work of the authors [8], [9], [10] that uncovered a new class of networks that can be analyzed robustly for any monotone kinetics. The next section reviews the required background on CRNs. Section 3 , presents the concepts of robust Lyapunov functions and graphically stable networks. Section 4, presents construction algorithms for these functions. Finally, section 5 presents biochemical applications of the proposed techniques.

M. Ali Al-Radhawi is with the Department of Mechanical Engineering, Massachusetts Institute of Technology, 77 Mass. Av., Cambridge, MA 02139, United States. Email: malirdwi@mit.edu.

D. Angeli is with the Department of Electrical \& Electronic Engineering, Imperial College London, London SW7 2AZ, UK. He is also with Dipartimento di Ingegneria dell'Informazione, University of Florence, 50139 Florence, Italy. Email: d.angeli@imperial.ac.uk.

\section{BACKGROUND ON CHEMICAL REACTION NETWORKS}

The field of CRN dynamics has an established literature [5], [2]. We review here the relevant background.

A reaction network has two mathematical elements: the stoichiometry and the kinetics. Stoichiometry describes the relative number of molecules of reactants and products between the sides of each reaction, while kinetics is concerned with the relations that govern the velocity of transformation of reactants into products. We explain both below.

\section{A. Stoichiometry}

A Chemical Reaction Network (CRN) is defined by a set of species $\mathscr{S}=\left\{X_{1}, . ., X_{n}\right\}$, and a set of reactions $\mathscr{R}=$ $\left\{\mathbf{R}_{1}, \ldots, \mathbf{R}_{\nu}\right\}$. Each reaction is denoted as:

$$
\mathbf{R}_{j}: \quad \sum_{i=1}^{n} \alpha_{i j} X_{i} \longrightarrow \sum_{i=1}^{n} \beta_{i j} X_{i}, j=1, . ., \nu,
$$

where $\alpha_{i j}, \beta_{i j}$ are nonnegative integers called stoichiometry coefficients. The expression on the left-hand side is called the reactant complex, while the one on the right-hand side is called the product complex. The forward arrow refers to the idea that the transformation of reactants into products is only occurring in the direction of the arrow. If the transformation is occurring also in the opposite direction, the reaction is said to be reversible and its reverse is listed as a separate reaction. For convenience, the reverse reaction of $\mathbf{R}_{j}$ is denoted as $\mathbf{R}_{-j}$. Note that we allow reactant or product complex to be empty, though not simultaneously. This is used to model external inflows and outflows of the CRN.

An autocatalytic reaction is a reaction in which there exists a species which appears on both sides of the reaction simultaneously. If all reactions are non-autocatalytic, the network is termed non-autocatalytic.

The stoichiometry of a network can be summarized by arranging the coefficients in an augmented matrix $n \times 2 \nu$ as:

$$
\tilde{\Gamma}=[A \mid B], \text { where }[A]_{i j}=\alpha_{i j},[B]_{i j}=\beta_{i j} .
$$

The two matrices can be subtracted to yield an $n \times \nu$ matrix $\Gamma=\left[\begin{array}{ll}\gamma_{1}^{T} & . . \gamma_{n}^{T}\end{array}\right]^{T}$ called the stoichiometry matrix, which is defined as $\Gamma=B-A$, or element-wise as:

$$
[\Gamma]_{i j}=\beta_{i j}-\alpha_{i j}
$$

\section{B. Kinetics}

Assume we have an isothermal well-stirred chemical reactor; this implies that the species are distributed uniformly in the reactor. In order to study kinetics, a nonnegative number $x_{i}$ is associated to each species $X_{i}$ to denote its 
concentration. Assume that the chemical reaction $\mathbf{R}_{j}$ takes place continuously in time. A reaction rate or velocity function $R_{j}: \overline{\mathbb{R}}_{+}^{n} \rightarrow \overline{\mathbb{R}}_{+}$is assigned to each reaction.

A widely-used expression which originates from statistical thermodynamics is given as:

$$
R_{j}(x)=k_{j} \prod_{i=1}^{n} x_{i}^{\alpha_{i j}},
$$

(the so called Mass-Action kinetics), with the convention $0^{0}=1$, where $k_{j}, j=1, . ., m$ are positive numbers known as the kinetic constants, and are usually highly uncertain.

The Mass-Action model is not a universal model since there are other models which are popular in systems biology like the Michaelis-Menten model, and the Hill model.

In this work we will pursue "kinetics-independent" approach. More precisely, we assume that the reaction rate function of a CRN is unknown except for satisfying the following assumptions:

AK1. it is a $\mathscr{C}^{1}$ function, i.e. continuously differentiable;

AK2. $x_{i}=0 \Rightarrow R_{j}(x)=0$, for all $i$ and $j$ such that $\alpha_{i j}>0$; AK3. it is nondecreasing with respect to its reactants, i.e

$$
\frac{\partial R_{j}}{\partial x_{i}}(x)\left\{\begin{array}{ll}
\geq 0 & : \alpha_{i j}>0 \\
=0 & : \alpha_{i j}=0
\end{array} .\right.
$$

AK4. The inequality in (4) holds strictly for all $x \in \mathbb{R}_{+}^{n}$.

Reaction rate functions satisfying A1-A4 are called admissible. For a given stoichiometric matrices $A, B$, the set of admissible reactions is denoted by $\mathscr{K}_{A}$. A network family is the triple $\mathscr{N}_{A, B}=\left(\mathscr{S}, \mathscr{R}, \mathscr{K}_{A}\right)$.

Remark 1: The assumptions above imply the monotonic dependence of the reaction rate on the concentration of its reactants. This captures the basic intuition about the nature of a reaction since, as the concentration of reactants increases, the likelihood of collision between molecules increases, and hence the rate of the reaction.

Note that, in principle, a monotonically decreasing dependence on the reactants can also be accommodated to model inhibition.

Remark 2: It can be noted that if a reaction rate function is admissible, then the corresponding Jacobian $\partial R / \partial x$ will exhibit a certain zero sign-pattern that can be read from the graph, and any matrix satisfying that pattern correspond to an admissible $R$.

\section{Dynamics}

The dynamics of a CRN with $n$ species and $\nu$ reactions are described by a system of ordinary differential equations (ODEs) as:

$$
\dot{x}(t)=\Gamma R(x(t)), x(0) \in \overline{\mathbb{R}}_{+}^{n}
$$

where $x(t)$ is the concentration vector evolving in the nonnegative orthant $\overline{\mathbb{R}}_{+}^{n}, \Gamma \in \mathbb{R}^{n \times \nu}$ is the stoichiometry matrix, $R(x(t))=\left[R_{1}(x(t)), R_{2}(x(t)), \ldots, R_{\nu}(x(t))\right]^{T} \in \overline{\mathbb{R}}_{+}^{\nu}$ is the reaction rates vector.

Note that (5) belongs to the class of positive systems, i.e, $\overline{\mathbb{R}}_{+}^{n}$ is forward invariant. In addition, the manifold $\mathscr{C}_{x_{\circ}}:=$

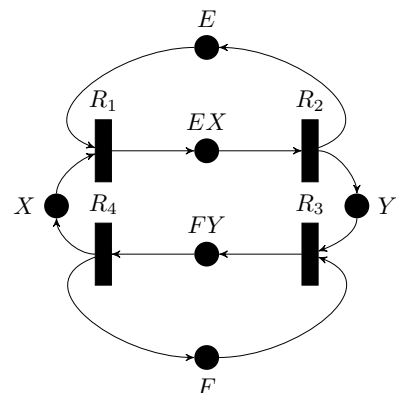

Fig. 1. Simplied Phoshophorylation-Dephosphorylation Cycle

$\left(\left\{x_{\circ}\right\}+\operatorname{Im}(\Gamma)\right) \cap \overline{\mathbb{R}}_{+}^{n}$ is forward invariant, and it is called the stoichiometric compatibility class associated with $x_{\circ}$. Therefore, all stability results in this paper are relative to the stoichiometry compatibility class.

A left null vector $d \in \mathbb{R}^{n}, d^{T} \Gamma=0$ with $d>0$ is said to be a conservation law. If there exists a conservation law $d \gg 0$, the network is said to be conservative; this implies that all stoichiometric classes are compact convex polyhedral sets.

The stoichiometry of the network will be assumed to satisfy the following assumptions:

AS1 There exists $v \in \operatorname{ker} \Gamma$ such that $v \gg 0$. This condition is necessary for the existence of a steady state in which all concentrations are positive.

Remark 3: AS1 is not essential for our methods, however, imposing it simplifies many of the results. Analysis of cases where it is not satisfied is discussed in [10].

\section{Graphical Representation and An Illustrative Example}

CRNs admit graphical representations naturally. A CRN can be modelled as a bipartite weighted directed graph or a Petri-Net. Then, the stoichiometry matrix $\Gamma$ becomes the incidence matrix of the graph.

As in illustration consider a common network which is the enzymatic futile cycle which is known as the Phoshophorylation-Dephosphorylation Cycle [11]. A simplified model can be written as:

$$
\begin{aligned}
& X+E \stackrel{\mathbf{R}_{1}}{\longrightarrow} E X \stackrel{\mathbf{R}_{2}}{\longrightarrow} Y+E, \\
& Y+F \stackrel{\mathbf{R}_{3}}{\longrightarrow} F Y \stackrel{\mathbf{R}_{4}}{\longrightarrow} X+F,
\end{aligned}
$$

$X$ represents the base substrate, $E$ is called a kinase which adds a phosphate group to $X$ to produce $Y$. This process is called phosphorylation. The dephosphorylation reaction is achieved by a phosphatase $F$ that removes the phosphate group from $Y$ to produce $X$. A more complete model will include the reverse of reactions $\mathbf{R}_{1}, \mathbf{R}_{3}$.

The petri-net representation is depicted in Figure 2. The corresponding ODE can be written as:

$$
\dot{x}=\left[\begin{array}{rrrr}
-1 & 0 & 0 & 1 \\
0 & 1 & -1 & 0 \\
1 & -1 & 0 & 0 \\
0 & 0 & 1 & -1 \\
-1 & 1 & 0 & 0 \\
0 & 0 & -1 & 1
\end{array}\right]\left[\begin{array}{c}
R_{1}\left(x_{2}, x_{5}\right) \\
R_{2}\left(x_{3}\right) \\
R_{3}\left(x_{2}, x_{6}\right) \\
R_{4}\left(x_{4}\right)
\end{array}\right]
$$




\section{ROBUST LYAPUNOV FUNCTIONS}

\section{A. A Motivating Example}

Consider the network presented at the end of the previous section. An intuitive way to analyze the network above is to consider the main loop in Figure 1 and study the sum of absolute reaction differences along the loop. This can be loosely paralleled to considering reactions as potentials, and noting that the difference of "potentials" causes the concentration of species to change. Hence, consider the following function:

$$
\begin{aligned}
V(x)= & \left|R_{1}(x)-R_{2}(x)\right|+\left|R_{2}(x)-R_{3}(x)\right| \\
& +\left|R_{3}(x)-R_{4}(x)\right|+\left|R_{4}(x)-R_{1}(x)\right| \\
= & \left|\dot{x}_{3}\right|+\left|\dot{x}_{2}\right|+\left|\dot{x}_{4}\right|+\left|\dot{x}_{1}\right|,
\end{aligned}
$$

which a piecewise linear in rates (PWLR) function. Hence, it can be analyzed region-wise. Consider for instance the region $\mathcal{W}=\left\{R_{1}(x) \geq R_{2}(x), R_{3}(x) \geq R_{2}(x), R_{3}(x) \geq\right.$ $\left.R_{4}(x), R_{4}(x) \geq R_{1}(x)\right\}$. Then, over that region the function can be written as:

$$
\frac{1}{2} V(x)=R_{1}(x)-R_{4}(x)+R_{3}(x)-R_{2}(x)
$$

Note that in the given region, the sign of $\dot{x}$ is determined and it can be read from the graph as follows: $\dot{x}_{1}, \dot{x}_{2}, \dot{x}_{5}, \dot{x}_{6}<$ 0 and $\dot{x}_{3}, \dot{x}_{4}>0$. By noting these signs are matched to the coefficients of $R(x)$ in (8) and the Jacobian of $R$ is nonnegative, we can write the following inequality in $\mathcal{W}^{\circ}$ :

$$
\begin{aligned}
\frac{1}{2} \dot{V}(x)= & \frac{\partial R_{1}}{\partial x_{5}} \dot{\bar{x}}_{5}+\frac{\partial R_{1}}{\partial x_{1}} \dot{x}_{1}-\frac{\partial R_{4}}{\partial x_{4}} \dot{x}_{4}^{+} \\
& +\frac{\partial R_{3}}{\partial x_{2}} \dot{x}_{2}+\frac{\partial R_{3}}{\partial x_{6}} \bar{x}_{6}-\frac{\partial R_{3}}{\partial x_{3}} \dot{x}_{3} \leq 0
\end{aligned}
$$

where the sign of the rate of change of each concentration is indicate above it.

Therefore, $\operatorname{sgn} \dot{V}$ can be determined conclusively without knowing the kinetics. In fact, this can be repeated for all regions to conclude that $V$ is decreasing along the trajectories of (6) for any admissible $R$.

The lesson that can be taken from this example is that a robust analysis of reaction networks can be carried out in the reaction coordinates. In addition, this analysis can be carried out by the utilization of PWLR functions that are defined in such manner that the sign pattern of $\dot{x}$ can be determined in each region by region inequalities, the signs of the coefficients of $R(x)$ are matched to the sign pattern, and the function can be patched continuously over the regions. The next sections will present a systematic generalization of this approach.

\section{B. Robust Lyapunov Functions: Reaction Coordinates}

Informally, a Robust Lyapunov Function (RLF) for a CRN is a function that can be determined solely based on the graphical structure of the network and that is decreasing for all admissible reaction rate functions. For instance, the function (7) can be considered as an RLF.

It is assumed that an RLF can be written as a composition $V=\tilde{V} \circ R$. As will be seen afterwards, the function $\tilde{V}$ used is invariant with respect to choice of the reaction rate function in $\mathscr{K}_{A}$. Hence, a formal definition can be stated as follows:

Definition 1 (Robust Lyapunov Function): Given (5). Let $\tilde{V}: \overline{\mathbb{R}}^{\nu} \rightarrow \overline{\mathbb{R}}_{+}$be locally Lipschitz. Then, $\tilde{V}$ is said to induce a Robust Lyapunov Function (RLF) with respect to the network family $\mathscr{N}_{A, B}$ if for any choice of $R \in \mathscr{K}_{A}$, the parameterized function $V_{R}=\tilde{V} \circ R$ is,

1) Positive-Definite: $V(x) \geq 0$, and $V(x)=0$ if and only if $R(x) \in \operatorname{ker} \Gamma$.

2) Nonincreasing: $\dot{V}(x) \leq 0$ for all $x \in \mathscr{C}_{x_{e}}$.

A network for which an RLF exists is terms a Graphically Stable Network (GSN).

The application of Lyapunov's second method gives the following theorem:

Theorem 1 (Lyapunov's Second Method): Given (5) with initial condition $x_{\circ} \in \mathbb{R}_{+}^{n}$, and let $\mathscr{C}_{x_{\circ}}$ as the associated stoichiometric compatibility class. Assume there exists an RLF Lyapunov function. and suppose that $x(t)$ is bounded,

1) then the equilibrium set $E_{x_{\circ}}$ is Lyapunov stable.

2) If, in addition, $V$ satisfies the LaSalle's Condition, then $x(t) \rightarrow E_{x_{\circ}}$ as $t \rightarrow \infty$ (i.e., the point to set distance of $x(t)$ to $E_{x_{\circ}}$ tends to 0 ).

3) If $V$ satisfies the LaSalle's condition, and all the trajectories are bounded, then: If there exists $x^{*} \in E_{x_{\circ}}$, which is isolated relative to $\mathscr{C}_{x_{\circ}}$ then it is a unique globally asymptotically stable equilibrium relative to $\mathscr{C}_{x_{0}}$.

Remark 4: Theorem 1 implies that the existence of two or more isolated equilibria, even if the interior's equilibrium is unique, excludes the possibility of the existence of an RLF which satisfies the LaSalle's condition. This is to be contrasted with deficiency-zero theorem [5] where boundary equilibria can be accommodated.

\section{Uncertain System Interpretation}

As arbitrary monotone kinetics are allowed in our formulation of the CRN family $\mathscr{N}_{A, B}$, the system (5) with kinetics $\mathscr{K}_{A}$ can be viewed as an uncertain system. In this subsection, we show that the Lyapunov function introduced above can be interpreted by shifting the analysis of the system to reaction coordinates. This enables us to view the network as a linear parameter varying system and the the RLF as a common Lyapunov function for the associated differential inclusion.

Let $r(t):=R(x(t))$, then we have:

$$
\dot{r}(t)=\frac{\partial R}{\partial x}(x(t)) \Gamma r(t)=\rho(t) \Gamma r(t),
$$

where $\rho(t):=\frac{\partial R}{\partial x}(x(t))$. We can write $\rho(t)$ as a conic combination of individual partial derivatives as follows:

$$
\frac{\partial R}{\partial x}(x(t))=\rho(t)=\sum_{i, j: \alpha_{i j}>0} \rho_{j i}(t) E_{j i},
$$

where $[\rho(t)]_{j i}=\rho_{j i}(t)$, and $\left[E_{j i}\right]_{j^{\prime} i^{\prime}}=1$ if $\left(j^{\prime}, i^{\prime}\right)=(j, i)$ and zero otherwise.

Let $s$ denote the number of elements in the support of 
$\partial R / \partial x$, and let $\kappa:\{1, . ., s\} \rightarrow\left\{(i, j): \alpha_{i j}>0\right\}$ be an indexing map. Then, we can write (9) as:

$$
\dot{r}=\sum_{i, j: \alpha_{i j}>0} \rho_{j i}(t) E_{j i} \Gamma r=\sum_{\ell=1}^{s} \rho_{\ell}(t) \Gamma^{\ell} r
$$

where $\Gamma^{\ell}=e_{j} \gamma_{i}^{T}, \rho_{\ell}(t)=\rho_{j i}(t)$, with $(i, j)=\kappa(\ell)$, and $\left\{e_{j}\right\}_{j=1}^{\nu}$ denotes the canonical basis of $\mathbb{R}^{\nu}$. Hence, eq. (11) represents a linear parameter-varying system which has $s$ nonnegative time-varying parameters $\left\{\rho_{1}(t), . ., \rho_{s}(t)\right\}$ where the system matrix belongs to the conic hull of the set of rankone matrices $\left\{\Gamma^{1}, \ldots, \Gamma^{s}\right\}$.

Hence, we have the following definition:

Definition 2 (Common Lyapunov Function): A function $\tilde{V}: \overline{\mathbb{R}}_{+}^{\nu} \rightarrow \overline{\mathbb{R}}_{+}$is said to be a Lyapunov function for the linear system $\dot{r}=\Gamma^{\ell} r$ if it is locally Lipschitz, nonnegative, has a negative semi-definite time-derivative, and $\operatorname{ker} \tilde{V} \subset$ $\operatorname{ker} \Gamma^{\ell}$. Furthermore, $\tilde{V}$ is said to be a common Lyapunov function for the set of linear systems $\left\{\dot{r}=\Gamma^{1} r, \ldots, \dot{r}=\Gamma^{s} r\right\}$ if it is a Lyapunov function for each of them, and $\operatorname{ker} \tilde{V}=$ $\bigcap_{\ell=1}^{s} \operatorname{ker} \Gamma^{\ell}$.

Hence, the characterization of Robust Lyapunov Functions in term of common Lyapunov functions can be stated as follows:

Theorem 2 (Common Lyapunov Function): Given the system (5). There exists a common Lyapunov function $\tilde{V}: \overline{\mathbb{R}}_{+}^{\nu} \rightarrow \overline{\mathbb{R}}_{+}$for the set of linear systems $\left\{\dot{r}=\Gamma^{1} r, \ldots, \dot{r}=\Gamma^{s} r\right\}$, if and only if $(\tilde{V}, R)$ induces the Robust Lyapunov function parameterized as $V_{R}(x)=\tilde{V}(R(x))$ for the $\mathrm{CRN}$ family $\mathscr{N}_{A, B}$.

Remark 5: An alternative change of variable can be given in terms of the extent of reaction $\xi$ which is defined as $\xi(t)=\int_{0}^{t} R(x(\tau)) d \tau$. This produces an alternative ODE representation for (5) which is given by $\dot{\xi}=R\left(x_{\circ}+\right.$ $\Gamma \xi, \xi(0)=0$. Hence, the reaction coordinate representation (9) can be interpreted more succinctly in terms of variational system $\dot{\delta \xi}=\frac{\partial R}{\partial x} \Gamma \delta \xi$. More details can be found in [9].

Remark 6: The results above can be interpreted in the species coordinates by defining a dual RLF which can be written as $V(x)=\hat{V}\left(x-x_{e}\right)$, where $x_{e}$ is an equilibrium for (5). However, this function is decreasing only in the stoichiometric class corresponding to $x_{e}$. More details can be found in [9].

\section{Piecewise Linear in Rates Lyapunov Functions}

In the previous subsection we have shown that the RLF we defined can be characterized in terms of a common Lyapunov function of an appropriate differential inclusion in reactioncoordinates. In fact, it is known the asymptotic stability of linear differential inclusions can be characterized in terms of the existence of a piecewise linear Lyapunov function [12]. Hence, in this section we define the class of piecewise linear in rates (PWLR) Lyapunov functions.

Consider a continuous Piecewise Linear (PWL) function $\tilde{V}$ defined over a polyhedral conic partition of $\mathbb{R}^{\nu}$. The partition is generated by a matrix $H \in \mathbb{R}^{p \times \nu}$, which is assumed to have some vector $\mu \in \operatorname{ker} H$ with $\mu \gg 0$, and does not have zero rows. Let $\Sigma_{1}, \ldots, \Sigma_{2^{p}}$ be the set of $p \times p$ signature matrices, i.e. all possible $\{ \pm 1\}$-diagonal matrices of size $p \times$ p. Define cones $\mathcal{W}_{1}, \ldots, \mathcal{W}_{2^{p}}$ as:

$$
\mathcal{W}_{k}=\left\{r \in \mathbb{R}^{\nu}: \Sigma_{k} H r \geq 0\right\} .
$$

As some of the intersections may have empty interiors, i.e., conflicting inequalities, we reorder the cones' indices such that the first $m$ cones are the nonempty-interior cones, i.e. $\mathcal{W}_{k}^{\circ} \neq \varnothing$ iff $k \in\{1, \ldots, m\}$.

We are ready to define the function:

Definition 3: Let $H$ with $\operatorname{ker} H=\operatorname{ker} \Gamma$, and $\left\{\mathcal{W}_{k}\right\}_{k=1}^{m}$ be defined as above, and assume that $C=\left[\begin{array}{lll}c_{1}^{T} & . & c_{m / 2}^{T}\end{array}\right]^{T} \in$ $\mathbb{R}^{m / 2 \times \nu}$ be the coefficients matrix. Then, $V: \mathbb{R}^{n} \rightarrow \mathbb{R}$ is said to be a Piecewise Linear in Rates (PWLR) function if it admits the representation $V(x)=\tilde{V}(R(x))$, where $\tilde{V}$ : $\mathbb{R}^{\nu} \rightarrow \mathbb{R}$ is a continuous PWL function given as

$$
\tilde{V}(r)=\left|c_{k}^{T} r\right|, r \in \mathcal{W}_{k} \cap-\mathcal{W}_{k}, k=1, . ., m / 2 .
$$

Note that by definition, if $\tilde{V}(r)=c_{k}^{T} r$, then the function is defined over the region $\mathcal{W}_{k}$, and if $\tilde{V}(r)=-c_{k}^{T} r$, then the corresponding region is $\mathcal{W}_{-k}:=\mathcal{W}_{m-k+1}=-\mathcal{W}_{k}$.

Within the class of PWLR functions, the subclass of convex PWLR functions admits a simpler representation:

Definition 4: Let $C=\left[\begin{array}{lll}c_{1}^{T} & . . & c_{m / 2}^{T}\end{array}\right]^{T} \in \mathbb{R}^{m / 2 \times \nu}$ be given such that there exists $v \in \operatorname{ker} C$ with $v \gg 0$. Then, $V$ : $\mathbb{R}^{n} \rightarrow \mathbb{R}$ is said to be a convex PWLR function if it admits the representation $V(x)=\tilde{V}(R(x))$, where $\tilde{V}: \mathbb{R}^{\nu} \rightarrow \mathbb{R}$ is a convex PWL given by

$$
\tilde{V}(r)=\max _{1 \leq k \leq m / 2}\left|c_{k}^{T} r\right|=\|C r\|_{\infty} .
$$

Remark 7: Verifying that a candidate PWLR function is an RLF can be formulated as a linear program. Furthermore, the LaSalle's condition can be verified also by a graphical algorithm. For more details, refer to [8].

\section{CONSTRUCTION OF ROBUST LYAPUNOV FUNCTIONS}

\section{A. A Sign Pattern Characterization}

In this section, we show that there is a set of sign constraints that the coefficients of a PWLR function needs to satisfy in order to be nondecreasing. These constraints will allow us to devise algorithm to construct PWLR Lyapunov functions.

As the motivating example may indicate, it can be shown that the nonpositivity of every term in the expansion of $\dot{V}$ is needed for it to be an RLF [8]. In order to verify this, it can be assumed, without loss of generality, that polyhedral partition (12) have the property that $\operatorname{sgn} \dot{x}$ is constant in the interior of each region. Hence, we can define signs $\sigma_{k 1}, \ldots, \sigma_{k n}$ to indicate sgn $\dot{x}$ in the region $\mathcal{W}_{k}$.

That can be used to define sign pattern vectors $b_{k 1} \ldots b_{k \nu}$, $k=1, . ., m_{s} / 2$, where:

$b_{k j}=\left\{\begin{aligned} \infty, & \text { if } \mathbf{R}_{j} \text { is an inflow } \\ 0, & \text { if } \exists i_{1}, i_{2} \in M_{j} \text { such that } \sigma_{k i_{1}} \sigma_{k i_{2}}<0, \\ -\sigma_{j i^{*}}, & \text { otherwise, for any } X_{i^{*}} \text { reactant of } \mathbf{R}_{j} .\end{aligned}\right.$

This enables us to state the following result: 
Theorem 3 (Sign Condition): Given a PWLR function $V$ defined by (13). The function is nondecreasing for any admissible $R$ if and only if the coefficient vectors $c_{k}, k=$ $1, \ldots, m$ satisfy for $\ell \in\{1, \ldots, n\} \backslash \mathcal{I}$, where $\mathcal{I}$ are the inflows, the following equality and inequality constraints.

$$
\begin{cases}c_{k j}=0 & \text { if } b_{q_{k} j}=0 \\ c_{k j} b_{q_{k} j} \geq 0 & \text { if } 0<\left|b_{q_{k} j}\right|<\infty\end{cases}
$$

\section{B. Construction Via Linear Programs}

Assume that the partition generator $H$ is fixed, hence $\left\{\mathcal{W}_{k}\right\}_{k=1}^{m}$ is determined. Nonnegativity can be verified via a linear constraint derived from Farkas' Lemma. For continuity, it is enough to verify it in a region $k$ with respect to its neighboring regions $\mathcal{N}_{k}$ which are all the regions in which they differ from $\mathcal{W}_{k}$ by the switching of one inequality only. The nondecreasingness condition can be verified using the sign characterization above. Hence, the following is stated:

Theorem 4: Consider (5), with $H,\left\{\Sigma_{k}\right\}_{k=1}^{m},\left\{B_{k}\right\}_{k=1}^{m}$ given as above. Consider the following linear program:

Find $\quad c_{k}, \xi_{k}, \zeta_{k} \in \mathbb{R}^{\nu}, \eta_{k j} \in \mathbb{R}, k=1, . ., \frac{m}{2} ; j \in \mathcal{N}_{k}$, subject to $c_{k}^{T}=\xi_{k}^{T} \Sigma_{k} H$, [Nonnegativity]

$$
\begin{aligned}
& c_{k}^{T}=\zeta_{k}^{T} \operatorname{diag}\left(b_{k}\right), \text { [Nondecreasingness] } \\
& c_{k}-c_{j}=\eta_{k j} \sigma_{k s_{k j}} h_{s_{k j}}, \text { [Continuety] } \\
& \xi_{k} \geq 0, \mathbf{1}^{T} \xi_{k}>0, \\
& \zeta_{k j} \geq 0, j \in\{1, . ., \nu\} \backslash \mathcal{I},
\end{aligned}
$$

where $s_{k j}$ is the index of the switched inequality. Then there exists a PWLR Lyapunov function with partitioning matrix $H$ if and only if there exist feasible solution to the above linear program with $\operatorname{ker} C=\operatorname{ker} \Gamma$. Furthermore, the PWLR function can be made convex by adding the constraints $\eta_{k j} \geq$ 0 .

A natural candidate for the partition matrix is $H=\Gamma$. Hence, we can write

$V(x)=c_{k}^{T} R(x)=\xi_{k}^{T} \Sigma_{k} \Gamma R(x)=\left\|\operatorname{diag}\left(\xi_{k}\right) \dot{x}\right\|_{1}, R(x) \in \mathcal{W}_{k}$.

If we have additional constraint that for all $k, \xi_{k}=\mathbf{1}$, then the Lyapunov function considered in [6],

$$
V(x)=\|\dot{x}\|_{1},
$$

can be recovered as a special case. However, there are classes of networks for which $H=\Gamma$ does not induce a PWLR Lyapunov function, while there exists a partitioning matrix $\hat{H}$ which does. Understanding when this happens is a challenging open question.

\section{Construction Via an Iterative Algorithm}

In this subsection, we present an iterative algorithm for constructing convex PWLR Lyapunov functions. The idea is to start with an initial PWLR function, and aim for restricting the active region of each linear function $c_{k}^{T} R(x(t))$ to the region for which it is nonincreasing on it, i.e $c_{k}^{T} \dot{R}(x(t)) \leq 0$. This is accomplished by adding extra linear components that satisfies certain properties.

Let $c_{1}$ be an initial coefficient vector. The active region of $c_{k}$ is $\mathcal{W}_{0}\left(c_{k}\right):=\left\{r:\|C r\|_{\infty}=c_{k}^{T} r\right\}$. The permissible region is $\mathcal{P}\left(c_{k}\right)=\left\{r: \operatorname{sgn}\left(c_{k i}\right) \gamma_{i}^{T} r \leq 0, i \in I_{k}\right\}$ where $I_{k}$ are the reactants of reactions that appear in $\operatorname{supp}\left(c_{k}\right)$. Note that this region is a subset of region in which $c_{k}^{T} \dot{R}(x) \leq 0$.

Note that in general, $\mathcal{W}_{0}\left(c_{k}\right) \not \subset \mathcal{P}\left(c_{k}\right)$. Therefore, we need to define a new PWL function with matrix $C_{1}$ so that $\mathcal{W}_{1}\left(c_{k}\right) \subset$ $\mathcal{P}\left(c_{k}\right)$. To achieve this, we augment new rows to $C_{0}:=c_{1}^{T}$. The new rows are of the form

$$
c_{m_{0}+i}:=c_{k}+\operatorname{sgn}\left(c_{k i}\right) \gamma_{i}, i \in I_{k}
$$

Thus, $C_{1}^{\prime}:=\left[\begin{array}{llll}C_{0}^{T} & c_{m_{0}+1} & \ldots & c_{m_{0}+n}\end{array}\right]^{T}$. Finally, $C_{1}$ is defined by eliminating linearly dependent pairs of rows from $C_{1}^{\prime}$.

Hence, Algorithm 1 can be described as:

1) Given $C_{0}=c_{1}^{T} \in \mathbb{R}^{\nu}$ with $\operatorname{ker} \Gamma \subset \operatorname{ker} C_{0}$. Set $k=1$.

2) Define $C_{k}^{\prime}:=\left[\begin{array}{llll}C_{k-1}^{T} & c_{m_{k}+1} & \ldots & c_{m_{k}+n}\end{array}\right]^{T}$, where $c_{m_{k}+i}:=c_{k}+\operatorname{sgn}\left(c_{k i}\right) \gamma_{i}, i=1, \ldots, n$.

3) Eliminate linearly dependent pairs of rows from $C_{k}^{\prime}$ with linearly dependent pairs of rows eliminated. If $C_{k}=C_{k-1}$ or $k>N$, stop.

4) Set $k:=k+1$, and go to step 2 ,

where $N$ is the maximum number of iterations allowed. Hence we can state:

Theorem 5: Consider (5). If Algorithm 1 terminates after finite number of iterations and $\operatorname{ker} C=\operatorname{ker} \Gamma$, then the resulting function is a PWLR Lyapunov function for the network.

\section{Construction Via Graphical Conditions}

It is possible to construct PWLR Lyapunov functions for CRNs with specific structure. We state the following result which enjoys having easy-to-check graphical condition:

Theorem 6: Consider an non-autocatalytic network with $\operatorname{dim}(\operatorname{ker} \Gamma)=1$. If, $\forall X_{i} \in \mathscr{S}$, there exists a unique output reaction, i.e every row in $\Gamma$ has a unique negative element, Then, the following is a PWLR function for the network:

$$
V(x)=\max _{1 \leq j \leq \nu} \frac{1}{v_{j}} R_{j}(x)-\min _{1 \leq j \leq \nu} \frac{1}{v_{j}} R_{j}(x),
$$

where $v=\left[v_{1} \ldots v_{\nu}\right]^{T} \in \operatorname{ker}(\Gamma), v \gg 0$.

Theorem 6 can be extended to allow the addition of the reverse of certain reactions. Note that adding the reverse of an irreversible reaction increases the dimension of the kernel of $\Gamma$ so that the original result would not normally apply.

Theorem 7: Consider the network $\mathscr{N}_{A, B}=\left(\mathscr{S}, \mathscr{R}, \mathscr{K}_{A}\right)$ which satisfies the conditions of Theorem 6 . Let $\mathscr{R}^{\prime} \subset \mathscr{R}$ be the set of reactions $\mathbf{R}_{j}$ that satisfy: if $\beta_{i j}>0$ and $\beta_{i k}>0$ then $j=k$. Equivalently, $\mathbf{R}_{j} \in \mathscr{R}^{\prime}$ if it is the only input reaction for all of its product species. Let $(\mathscr{S}, \tilde{\mathscr{R}})$ be the CRN constructed by adding reverse reactions for reactions belonging to $\mathscr{R}^{\prime}$. Then, the following is a PWLR RLF for the new network:

$$
\begin{aligned}
V(x)=\max _{1 \leq j \leq \nu} \frac{1}{v_{j}}( & \left.R_{j}(x)-\chi_{j} R_{-j}(x)\right)- \\
& \min _{1 \leq j \leq \nu} \frac{1}{v_{j}}\left(R_{j}(x)-\chi_{j} R_{-j}(x)\right),
\end{aligned}
$$

where $\chi_{j}=1$ if $\mathbf{R}_{\mathbf{j}} \in \tilde{\mathscr{R}} \backslash \mathscr{R}$, and $\chi_{j}=0$ otherwise. 


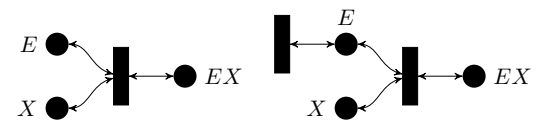

(a) Simple Binding (b) Simple Binding With Enzyme InflowOutflow

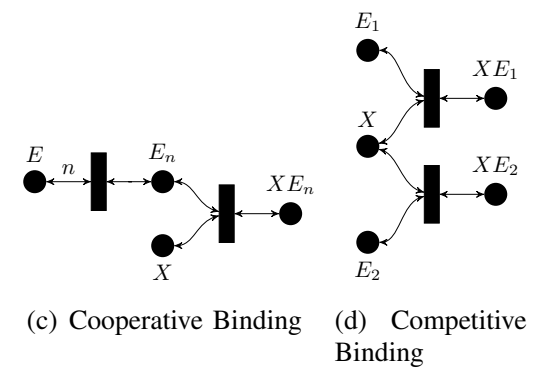

Fig. 2. Basic Biochemical Examples

\section{EXAMPLES}

Within the class of networks that satisfy the necessary conditions outlined in [8], [9], our proposed algorithms are reasonably successful. In most examples, either a PWLR function is constructed, or a necessary condition is violated rendering the Lyapunov function inadmissable. Nevertheless, there are networks which do not violate any necessary condition, and still our algorithms did not succeed in constructing a Lyapunov function. In this section, several examples of graphically stable biochemical networks are presented.

\section{A. Basic Motifs}

In this subsection, several simple biochemical networks [13] are presented. These networks are fairly simple and all of them can be analyzed using the deficiency zero theory in the case of Mass-Action kinetics. However, they presented here to show that the properties that our theory requires are obeyed by the basic biochemical motifs, which establishes its applicability and generality.

1) Simple Binding Reaction: Figure 2-a) represents a simple reversible binding reaction:

$$
X+E \rightleftharpoons E X
$$

The corresponding PWLR Lyapunov function can be found easily using Theorem 6 and is given by:

$$
V(x)=\left|R_{1}(x)-R_{2}(x)\right| .
$$

In general any reversible reaction: $\sum_{i=1}^{n} \alpha_{i} X_{i} \rightleftharpoons$ $\sum_{i=1}^{n} \beta_{i} X_{i}$, admits the same Lyapunov function.

2) Simple Binding With Enzyme Inflow-Outflow: Figure 2-b) represents the following binding reaction with enzyme inflow-outflow:

$$
X+E \underset{R_{-1}}{\stackrel{R_{1}}{\rightleftharpoons}} E X, 0 \underset{R_{-2}}{\stackrel{R_{2}}{\rightleftharpoons}} E
$$

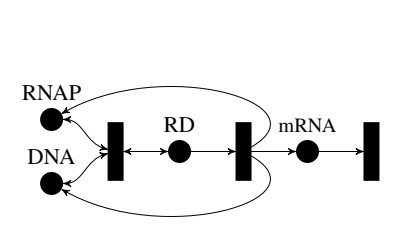

(a)

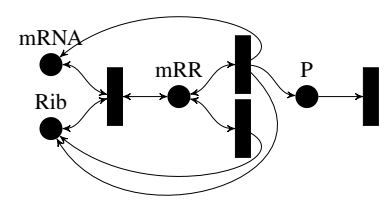

(b)
Fig. 3. Translation and Transcription

The corresponding PWLR Lyapunov function can be found using Theorem 7 and is given by:

$$
\begin{aligned}
V(x)= & \max \left\{R_{1}(x)-R_{-1}(x), R_{2}(x)-R_{-2}(x), 0\right\} \\
& -\min \left\{R_{1}(x)-R_{-1}(x), R_{2}(x)-R_{-2}(x), 0\right\} .
\end{aligned}
$$

The underlying networks for which Theorem 7 was applied is $0 \rightarrow E, 0 \rightarrow X, X+E \rightarrow X E, X E \rightarrow 0$.

3) Cooperative Binding Reaction: The following reaction represents the situation where $n$ enzyme molecules $E$ need to bind to each other to react to $X$, which is given in Figure 2-c). The case $n=2$ is called dimerization:

$$
n E \underset{R_{-1}}{\stackrel{R_{1}}{\rightleftharpoons}} E_{n}, E_{n}+X \underset{R_{-2}}{\stackrel{R_{2}}{\rightleftharpoons}} X E_{n}
$$

The corresponding PWLR Lyapunov function can be found using Theorem 7 and is given by (19). The underlying networks for which Theorem 7 was applied is $0 \rightarrow E, 0 \rightarrow$ $X, E \rightarrow E_{n}, E_{n}+X \rightarrow X E_{n}, X E_{n} \rightarrow 0$.

4) Competitive Binding Reaction: The following reaction describes the situation when two molecules $E_{1}, E_{2}$ compete to bind with $X$, which is given in Figure 2-d):

$$
E_{1}+X \underset{R_{-1}}{\stackrel{R_{1}}{\rightleftharpoons}} X E_{1}, X+E_{2} \underset{R_{-2}}{\stackrel{R_{2}}{\rightleftharpoons}} X E_{2}
$$

The corresponding PWLR Lyapunov function can be found using Theorem 7 and is given by (19). The underlying networks for which Theorem 7 was applied is $0 \rightarrow E_{1}, E_{1}+$ $X \rightarrow X E_{1} \rightarrow X E_{1} \rightarrow 0,0 \rightarrow X E_{2} \rightarrow X+E_{2}, E_{2} \rightarrow 0$.

\section{B. Transcription and Translation Networks}

1) Simplified Transcription Reaction: Figure 3-a) shows the the transcription network which describes the production of mRNA from DNA using the RNA polymerase [13]:

$$
\begin{gathered}
\mathrm{RNAP}+\mathrm{DNA} \underset{R_{-1}}{\stackrel{R_{1}}{\rightleftharpoons}} \mathrm{RD} \stackrel{R_{2}}{\longrightarrow} \mathrm{RNAP}+\mathrm{DNA}+\mathrm{mRNA} \\
\text { mRNA } \stackrel{R_{3}}{\longrightarrow} 0 .
\end{gathered}
$$

Applying Theorem 7 or Theorem 5, the following PWLR Lyapunov function can be found:

$$
\begin{aligned}
V(x)= & \max \left\{R_{1}(x)-R_{-1}(x), R_{2}(x), R_{3}(x)\right\}- \\
& \min \left\{R_{1}(x)-R_{-1}(x), R_{2}(x), R_{3}(x)\right\} .
\end{aligned}
$$

Alternatively, Theorem 4 can be used, and the Lyapunov function found can be written as:

$$
V(x)=\left\|\operatorname{diag}\left([1,1,1,3]^{T}\right) \dot{x}\right\|_{1},
$$


where the species are ordered as RNAP, DNA, RD, P.

Note this network has deficiency one, hence no information regarding stability can be inferred. Furthermore, the procedure proposed by [14] has been reported not to work if the network is considered as whole.

2) Simplified Translation Reaction With A Leak: Figure 3-b) shows the the translation network which describes the production of a protein from the translation of mRNA using the Ribosom [13]. However, the leaking of the RibosommRNA complex to a ribosom is also modelled:

$$
\begin{gathered}
\mathrm{Rib}+\mathrm{mRNA} \underset{R_{-1}}{\stackrel{R_{1}}{\rightleftharpoons}} \mathrm{mRR} \underset{R_{2}}{\longrightarrow} \mathrm{mRNA}+\mathrm{P}+\text { Ribo } \\
\mathrm{mRR} \stackrel{R_{4}}{\longrightarrow} \mathrm{Rib}, \mathrm{P} \stackrel{R_{4}}{\longrightarrow} 0 .
\end{gathered}
$$

Note that the kernel space of the stoichiometry matrix is spanned by $[1,1,0,0,1]^{T}$ and $[1,0,1,0,0]^{T}$. Hence, AS1 is not satisfied and all equilibria belong to the boundary. Note also, that the dynamic of other species are independent of the dynamics of $P$. Hence, the network can be consider as a cascade of Rib + mRNA $\rightleftharpoons \mathrm{mRR} \longrightarrow$ mRNA + Ribo, $\mathrm{mRR} \longrightarrow \mathrm{Rib}$ and $0 \longrightarrow P \longrightarrow 0$. Applying Theorem 4 or Theorem 5 for the first network we get the following Lyapunov function:

$$
\begin{gathered}
V_{1}(x)=\max \left\{R_{4}(x), R_{1}(x)-R_{2}(x)-R_{3}(x)-R_{4}(x),\right. \\
\left.-R_{1}(x)+R_{2}(x)+R_{3}(x)\right\} .
\end{gathered}
$$

The LaSalle's condition can also verified to establish that $\dot{V}(x)=0$ implies $\dot{x}=0$.

Note that it is convex, but lacks symmetry, and can not be written using the $\ell_{\infty}$ notation. Nevertheless, it can be verified that it is nonnegative and vanishes exactly on the equilibrium set. The second network can be analyzed using this Lyapunov function:

$$
V_{2}(x)=\left|R_{3}(x)-R_{4}(x)\right| .
$$

Stability can be established using a CICS argument [15].

\section{Phosphorylation-Dephosphrylation Cycles}

The futile cycle or the phosphorylation-dephosphorylation cycle [11] has been analyzed as a motivating example. This kind of cycle appears frequently in biochemical networks, and can be interconnected in several way; we discuss some here.

1) Multiple Futile Cycles With Distinct Enzymes: Consider a cascade of $n$ futile cycle as shown in Figure 4-a). The associated reaction network for the case $n=2$ is given as:

$$
\begin{aligned}
& X_{0}+E_{0} \underset{k_{-1}}{\stackrel{k_{1}}{\leftrightarrows}} E_{0} X_{0} \stackrel{k_{2}}{\longrightarrow} X_{1}+E_{0}, \\
& X_{1}+F_{0} \underset{k_{-3}}{\stackrel{k_{3}}{\leftrightarrows}} F_{0} X_{1} \stackrel{k_{4}}{\longrightarrow} X_{0}+F_{0}, \\
& X_{1}+E_{1} \underset{k_{-5}}{\stackrel{k_{5}}{\leftrightarrows}} E_{1} X_{1} \stackrel{k_{6}}{\longrightarrow} X_{2}+E_{1}, \\
& X_{2}+F_{1} \underset{k_{-7}}{\stackrel{k_{7}}{\leftrightarrows}} F_{1} X_{2} \stackrel{k_{8}}{\longrightarrow} X_{1}+F_{1} .
\end{aligned}
$$

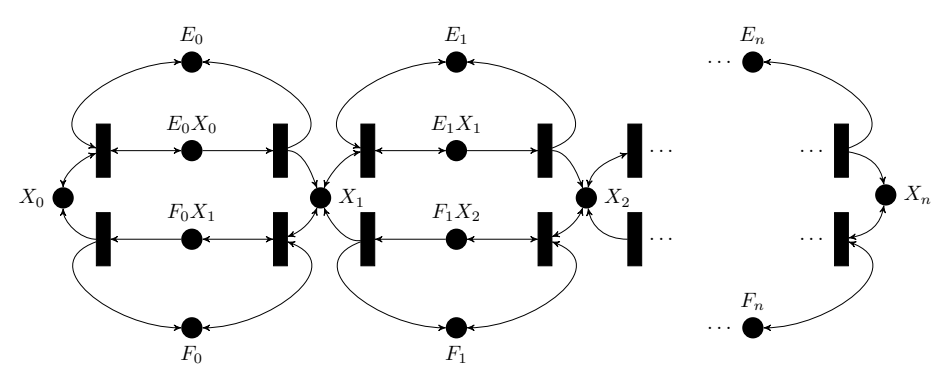

(a) Cascade of Multiple Futile Cycles with distinct enzymes

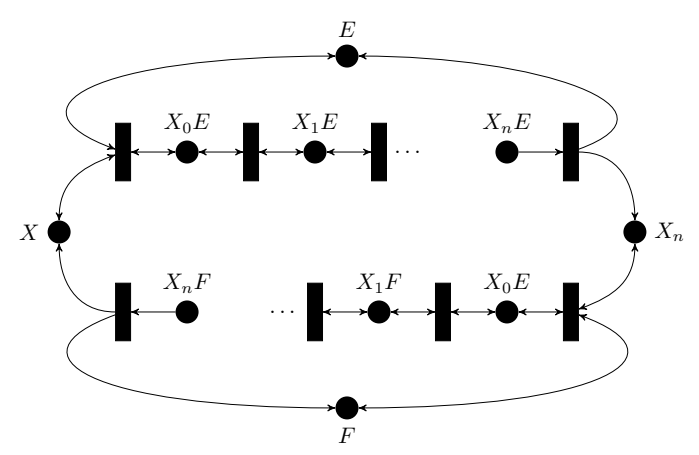

(b) Multiple Futile Cycle with a processive mechanism

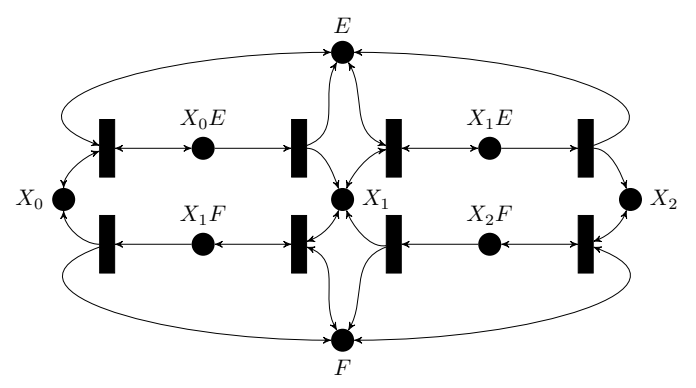

(c) Double Futile Cycle with a distributive mechanism

Fig. 4. Several Types of Cascades of Futile Cycles.

Both Theorems 4, 5 are applicable. A PWLR Lyapunov function for the $n$ cascade can be represented as:

$$
V(x)=\|\operatorname{diag}(\xi) \dot{x}\|_{1}
$$

with $\xi=[2,2, \ldots, 2,1,1, \ldots, 1]^{T}$ with the ordering as $X_{0}, \ldots, X_{n}, E_{0}, E_{1}, \ldots, F_{n-1} X_{n}$.

This network has deficiency 2 , hence the zero-deficiency theorem will not apply. Also, the results of [7] can not be applied since $X_{1}$ is adjacent to more than two reactions.

2) Multiple Futile Cycles With Processive Mechanism:

Figure 4-b) depicts a multiple-site futile cycle with a processive mechanism [16]. The reaction network can be written as:

$$
\begin{aligned}
& X_{0}+E \rightleftharpoons E X_{0} \rightleftharpoons E X_{1} \rightleftharpoons \ldots \rightleftharpoons E X_{n} \longrightarrow X_{n}+E, \\
& X_{n}+F \rightleftharpoons F X_{n} \rightleftharpoons \ldots \rightleftharpoons E X_{1} \rightleftharpoons E X_{0} \longrightarrow X_{0}+F,
\end{aligned}
$$

It can be noticed that for every $n$, the network satisfies the graphical conditions of Theorem 5. Therefore, the Lyapunov function can be written as follows:

$$
V(x)=\max _{k \neq j}\left(R_{k}(x)-R_{-k}(x)+R_{-j}(x)-R_{j}(x)\right),
$$


where $R_{-k}(x): \equiv 0$ if $\mathbf{R}_{k}$ is irreversible.

Hence, multiple degenerate equilibria are precluded. Since the network is conservative, it is persistent. This shows that any positive equilibrium is GAS. This result can be used as an alternative method to discard the above network above as a viable model for the first stage in the MAPK cascade [16]; since the later has been observed experimentally to accommodate multiple non-degenerate equilibria.

3) Double Futile Cycles With a Distributive Mechanism: Figure 4-c) describes a double futile cycle with a distributive mechanism [16], which is described by the following set of reactions:

$$
\begin{aligned}
& X_{0}+E \underset{k-1}{\stackrel{k_{1}}{\leftrightharpoons}} E X_{0} \stackrel{k_{2}}{\longrightarrow} X_{1}+E, \\
& X_{1}+F \underset{k_{-3}}{\stackrel{k_{3}}{\leftrightharpoons}} F X_{1} \stackrel{k_{4}}{\longrightarrow} X_{0}+F, \\
& X_{1}+E \underset{k_{-5}}{\stackrel{k_{5}}{\leftrightharpoons}} E X_{1} \stackrel{k_{6}}{\longrightarrow} X_{2}+E, \\
& X_{2}+F \underset{k_{-7}}{\stackrel{k_{7}}{\leftrightharpoons}} F X_{2} \stackrel{k_{8}}{\longrightarrow} X_{1}+F,
\end{aligned}
$$

It can be verified that the negative of the Jacobian of the network above has always strictly negative principle minor; for instance the minor corresponding to $X_{0}, X_{1}, X_{2}, E, F X_{1}, E X_{1}$ is negative for any choice of the kinetics. This violates a the result that the Jacobians of GSNs can not have negative principle minors [8]. This implies that it can not admit a PWLR Lyapunov function. Indeed, the above network is known to admit multiple nondegenerate equilibria some of which are unstable.

\section{T-Cell Kinetic Proofreading Network}

McKeithan [17] proposed a model of a ligand which is peptide-major histocompatibility complex $M$ binding to a $T$-cell receptor; the receptor-ligand complex undergoes several reactions to reach the final complex $C_{N}$. The chain of reactions increase sensitivity and hence it is called a kinetic proofreading process. Figure 5 depicts the reaction network, which is given by the following set of reactions:

$$
\begin{aligned}
T+M & \rightleftharpoons C_{0} \longrightarrow C_{1} \longrightarrow \ldots \longrightarrow C_{N} \\
C_{1} & \longrightarrow T+M, C_{2} \longrightarrow T+M, \ldots, C_{N} \longrightarrow T+M
\end{aligned}
$$

Applying Theorem 4, it can be shown that for any $N \geq 1$, the network admit the following PWLR Lyapunov function:

$$
V_{N}(x)=\left\|\operatorname{diag}\left([1,1,2,2, . ., 2]^{T}\right) \dot{x}\right\|_{1},
$$

where the species are ordered as $T, M, C_{0}, C_{1}, \ldots, C_{N}$. Note that this network does not meet the graphical requirements of Theorem 7. Using this, it can be shown that any positive equilibrium is unique and GAS.

The monotone-systems approach proposed by [7] is not applicable here since the species $T$ and $M$ are adjacent to more than two reactions. Nevertheless, [1] showed that this network is weakly reversible and zero-deficiency; therefore any positive equilibrium is unique relative to the interior and is locally asymptotically stable. In order to infer global

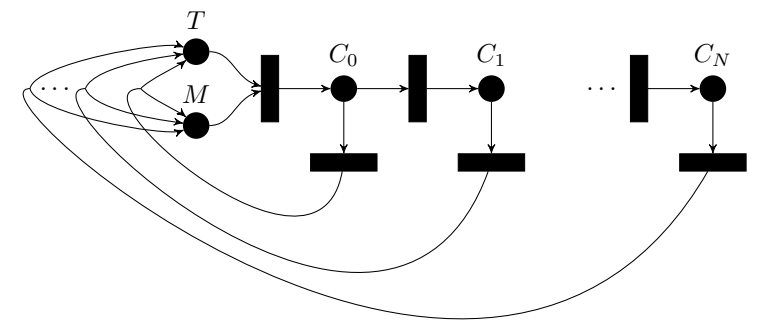

Fig. 5. McKeithan's network.

stability, it was needed to compute the equilibria explicitly to preclude the possibility of having a boundary equilibrium stoichiometrically compatible with a positive equilibrium. In comparison, our approach is more powerful, since the former approach is limited to Mass-Action kinetics, and can not infer global stability directly.

\section{REFERENCES}

[1] E. D. Sontag, "Structure and stability of certain chemical networks and applications to the kinetic proofreading model of T-cell receptor signal transduction," IEEE Transactions on Automatic Control, vol. 46, no. 7, pp. 1028-1047, 2001.

[2] D. Angeli, "A tutorial on chemical reaction network dynamics," European Journal of Control, vol. 15, no. 3-4, pp. 398-406, 2009.

[3] J. E. Bailey, "Complex biology with no parameters," Nature Biotechnology, vol. 19, no. 6, pp. 503-504, 2001.

[4] F. Horn and R. Jackson, "General mass action kinetics," Archive for Rational Mechanics and Analysis, vol. 47, no. 2, pp. 81-116, 1972.

[5] M. Feinberg, "The existence and uniqueness of steady states for a class of chemical reaction networks," Archive for Rational Mechanics and Analysis, vol. 132, no. 4, pp. 311-370, 1995.

[6] H. Maeda, S. Kodama, and Y. Ohta, "Asymptotic behavior of nonlinear compartmental systems: nonoscillation and stability," IEEE Transactions on Circuits and Systems, vol. 25, no. 6, pp. 372-378, 1978.

[7] D. Angeli, P. De Leenheer, and E. Sontag, "Graph-theoretic characterizations of monotonicity of chemical networks in reaction coordinates," Journal of mathematical biology, vol. 61, no. 4, pp. 581-616, 2010.

[8] M. A. Al-Radhawi and D. Angeli, "New approach for the stability of complex reaction networks: Piecewise linear in rates Lyapunov functions," IEEE Transactions on Automatic Control, To Appear, 2016.

[9] M. Ali Al-Radhawi and D. Angeli, "Robust Lyapunov functions for reaction networks: An uncertain system framework," arXiv:1509.02086, 2015.

[10] M. Ali Al-Radhawi, "New approach to the stability and control of reaction networks," Ph.D. dissertation, Imperial College London, 2015.

[11] M. Samoilov, S. Plyasunov, and A. P. Arkin, "Stochastic amplification and signaling in enzymatic futile cycles through noise-induced bistability with oscillations," Proceedings of the National Academy of Sciences of the United States of America, vol. 102, no. 7, pp. 2310$2315,2005$.

[12] A. P. Molchanov and Y. S. Pyatnitskiy, "Criteria of asymptotic stability of differential and difference inclusions encountered in control theory," Systems \& Control Letters, vol. 13, no. 1, pp. 59-64, 1989.

[13] D. Del Vecchio and R. M. Murray, Biomolecular Feedback Systems. Princeton University Press, 2014.

[14] F. Blanchini and G. Giordano, "Piecewise-linear Lyapunov functions for structural stability of biochemical networks," Automatica, vol. 50, no. 10 , pp. $2482-2493,2014$.

[15] E. D. Sontag, "A remark on the converging-input converging-state property," IEEE Transactions on Automatic Control, vol. 48, no. 2, pp. 313-314, 2003.

[16] C. Conradi, J. Saez-Rodriguez, E. D. Gilles, and J. Raisch, "Using chemical reaction network theory to discard a kinetic mechanism hypothesis," IEE Proceedings-Systems Biology, vol. 152, no. 4, pp. 243-248, 2005.

[17] T. W. Mckeithan, "Kinetic proofreading in T-cell receptor signal transduction," Proceedings of the national academy of sciences, vol. 92, no. 11, pp. 5042-5046, 1995. 